\title{
EDITORIAL
}

\section{UNA DEUDA EN LA SALUD DE CHILE: CONTAMINACIÓN DEL AIRE Y CAMBIO CLIMÁTICO}

CHILE'S HEALTH DEBT: AIR POLLUTION AND CLIMATE CHANGE

La contaminación del aire por material particulado y gases de origen antropogénico es uno de los temas ambientales que más directamente afectan a la población chilena. A pesar de los esfuerzos desplegados los últimos 30 años por los planes de descontaminación establecidos en los diversos centros urbanos e industriales de nuestro país, todavía se continúan excediendo las normas de calidad del aire, y sobre el 50\% de la población que vive en ellos está expuesta a niveles nocivos de contaminantes $(1,2)$. Peor aún, las normas primarias de calidad del aire vigentes no han incorporado contaminantes reconocidamente dañinos tales como el mercurio, benceno, y arsénico entre otros.

El efecto a corto y mediano plazo de los contaminantes está determinado fundamentalmente por la "dosis efectiva", producto de tres factores: tiempo de exposición, concentración del contaminante y ventilación minuto (1).

Entre los segmentos más vulnerables a la contaminación aérea y que, por lo tanto, son motivo de especial preocupación se encuentran los enfermos respiratorios y cardiovasculares como también las embarazadas y los grupos etarios extremos: senescentes y niños, especialmente los menores de cinco años (1).

Los artículos de revisión de este número de la revista analizan esta situación como también el impacto en la contaminación aérea de un fenómeno global como lo es el cambio climático y el de emergencias ambientales de aparición súbita y difíciles de controlar como lo son los incendios forestales.

Los niños son particularmente susceptibles a los efectos deletéreos de la contaminación del aire debido a diferencias anatómicas, fisiológicas y de estilos de vida. Entre estas desventajas se incluyen la menor efectividad de la tos, porque la musculatura respiratoria está aún en desarrollo y la mayor ventilación en reposo a expensas de la frecuencia respiratoria, lo cual aumenta la dosis efectiva de contaminantes. Además, la ausencia de ventilación colateral agrava la obstrucción de vías aéreas periféricas ( $<2 \mathrm{~mm}$ de diámetro), que a diferencia de los adultos genera el $50 \%$ de la resistencia total al flujo aéreo. Por otra parte los mecanismos defensivos no están aun plenamente desarrollados y es más dificultosa la eliminación de partículas que penetran en las vías aéreas. A todo esto, se suma que los niños pasan la mayor parte de su tiempo al aire libre, lo cual aumenta su exposición al aire atmosférico y sus contaminantes (3).

Uno de los efectos más frecuentes de la contaminación en los niños es el aumento de las infecciones respiratorias, especialmente virales (VRS, influenza), existiendo evidencia que ello se debería a modificaciones en el sistema inmune y en el microbioma humano. También se puede afectar el desarrollo neurológico, favorecer el desarrollo de patologías oncológicas y de enfermedades crónicas no trasmisibles como diabetes y asma bronquial, alteraciones cardiovasculares, malformaciones congénitas y salud mental (4).

Algunos contaminantes atmosféricos como dióxido de azufre, óxidos de nitrógeno y el material particulado, pueden aumentar los síntomas de asma bronquial y desencadenar exacerbaciones en niños asmáticos. También se ha detectado que exposición prolongada y precoz a contaminantes aéreos, especialmente los generados por el tráfico de vehículos motorizados, pueden aumentar el riesgo de desarrollar asma bronquial. Se ha llegado a estimar que un 13\% de los nuevos niños asmáticos pueden deberse a la contaminación atmosférica (5).

En niños expuestos a la contaminación del aire se ha comunicado disminución leve de CVF (10 mL/año) y de $\mathrm{VEF}_{1}(16 \mathrm{~mL} /$ año), lo que en la edad adulta podría llegar a tener importancia, ya que la exposición precoz a

Dr. Manuel Oyarzún G.

Profesor titular Instituto de Ciencias Biomédicas

Facultad de Medicina

Universidad de Chile

Email:manueloyarzun@uchile.cl
Dra. Patricia Matus C.

Profesora Asistente

Depto. Epidemiología y Estudios en Salud

Facultad de Medicina

Universidad de Los Andes

Email: pimatus@uandes.cl 
contaminantes atmosféricos podría significar menores valores espirométricos basales en la adultez y en el caso de desarrollar por ejemplo una enfermedad pulmonar obstructiva crónica (EPOC), tendrían de partida valores de función pulmonar inferiores a la población no expuesta a esta contaminación en su niñez (6).

Como una probable consecuencia del cambio climático, la exposición a emanaciones de incendios forestales es un problema creciente de salud pública tanto en Chile como en el resto del mundo. En el último decenio (20112020) en Chile se ha registrado un promedio anual de 6398 incendios que afectan anualmente a 122.328 ha, superficie que equivale a 1,9 veces el área de Santiago. La asociación entre la exposición a la contaminación de material particulado aéreo y gases derivados de incendios forestales y la morbilidad respiratoria por exacerbación de asma y enfermedades respiratorias crónicas, ha sido comunicada en numerosos estudios epidemiológicos. Los principales mecanismos de morbilidad serían el estrés oxidativo, la producción de citoquinas proinflamatorias, la activación endotelial y la disfunción del sistema nervioso autónomo. Si bien las consecuencias de la exposición a largo plazo y su eventual recuperación es muy poco conocida, existe evidencia de que en la población infantil los asmáticos son más susceptibles a la exposición a material particulado durante los incendios forestales, siendo más afectados los niños entre 0 a 4 años (OR= 8,3\% (IC 95\% 2,2-14,9)) y los obesos (7).

La contaminación atmosférica global ha provocado además de los efectos locales descritos, una alteración del estado del clima terrestre. El Cambio Climático consecuente y sus efectos, se pueden observar y cuantificar en el momento actual y no son un problema de las "futuras generaciones" como en algún tiempo se pensó. Chile no está exento del problema y existe evidencia de la carga de enfermedad y sufrimiento que esta condición global provoca en las comunidades (8).

Nuestro país, si bien ha mostrado algunos avances en materia de control y gestión de la calidad del aire, ha evidenciado retrocesos importantes, como es la aceptación fáctica de la existencia de "zonas de sacrificio", ejemplo los puertos de Huasco, en el norte Puchuncavíy Ventanas en la zona central y Coronel en la zona sur. En dichas áreas se han instalado una cantidad tal de fuentes industriales, que cumpliendo la normativa de emisión cada una de ellas, provocan en forma agregada un impacto permanente sobre la población. Este impacto no se ha visto contrapesado por acciones preventivas o de mitigación por parte de las autoridades sanitarias y ambientales que aseguren a dicha población gestionar su riesgo.

El diagnóstico precoz y tratamiento oportuno de alteraciones pulmonares, cardiovasculares y cognitivas, entre otras -especialmente en la población vulnerable- debiera ser una regla que no se observa. Tampoco existe claridad en el establecimiento de planes de adaptación al cambio climático, que ya es una realidad establecida en el país. Por lo tanto, los artículos sobre contaminación ambiental que se publican en este número de la revista, además de entregar información a la comunidad biomédica, tienen la intención de emitir una voz de alerta sobre el principal problema ambiental del país, la contaminación atmosférica, que aún no ha sido resuelto y se continúa complejizando con los efectos del Cambio Climático. El desarrollo de respuestas eficaces a estos problemas por parte de todos los estamentos de la sociedad es un imperativo que no se puede ni se debe continuar aplazando

REFERENCIAS

1. Oyarzún M. Situación de la relación ambiente y salud en Chile. Boletín Academia Chilena de Medicina 51(1): 133-142, 2014

2. Gobierno de Chile Ministerio del Medio Ambiente Tercer Informe del Estado del Medio ambiente (IEMA) 2020.Capítulo Cambio Climático [Internet]. Sistema Nacional de Información Ambiental (SINIA). 2021 [citado el 10.11.2021]. Disponible en: https://sinia. mma.gob.cl/index.php/datos-del-informe-delestado-del-medio-ambiente/

3. Oyarzún M. Contaminación atmosférica y sus efectos en salud Rev Chil Enferm Respir 2010; 26 (1): 16-25 http://dx.doi.org/10.4067/S071773482010000100004

4. Montes S. Contaminación ambiental e infecciones respiratorias en niños. Neumol. Pediatr. 2021; 16 (4): 161-163

5. Ubilla C, Yohannessen K. Contaminación atmosférica y asma en niños Neumol. Pediatr. 2021; 16 (4): 164-166.

6. Cai $Y$, Hansell AL, Granell R, Blangiardo M, Zottoli
M, Fecht D et al Prenatal, Early-Life, and Childhood Exposure to Air Pollution and Lung Function: The ALSPAC Cohort. Am J Respir Crit Care Med. 2020;202(1):112-123. doi: $10.1164 / \mathrm{rccm} .201902-$ $02860 \mathrm{C}$

7. Sandoval B, Rettig D, Reyes T, Oyarzún M. Impacto de la contaminación aérea por incendios forestales en la morbimortalidad de la población expuesta. Neumol. Pediatr. 2021; 16 (4): 167-171.

8. Matus P. Desafío del cambio climático y la contaminación del aire Neumol. Pediatr. 2021; 16 (4): 157-160 